\title{
CrysP: Multi-Faceted Activity-Infused Presence in Emerging Social Networks
}

\author{
Saguna $^{1}$ and Arkady Zaslavsky ${ }^{1,2}$ Dipanjan Chakraborty ${ }^{3}$ \\ 1 Monash University, Melbourne, Victoria, Australia \\ 2 Luleå University of Technology, SE-971 87, Luleå, Sweden, \\ 3 IBM Research, India Research Lab, New Delhi, India. \\ saguna. saguna@monash.edu, arkady.zaslavsky@ltu. se , cdipanjan@ibm.com,
}

\begin{abstract}
This paper presents Crystal Presence (CrysP) approach where we fuse presence and activity in the realm of social networking. CrysP functions on the basis of the activity-infused multi-faceted presence information. This information is derived from user's involvement in multiple activities at any point in time. We propose and develop the CrysP System (CrysPSys) to determine CrysP from the user and his/her environmental context information (mainly relating to user activity, moods/emotions and cognition/thoughts) and then sharing the relevant CrysP with the appropriate devices, services and social-networks (Corporate, Personal and Interest networks) and blogs (text, micro, video, photo and voice). We also highlight the benefits and challenges faced in developing such a system. This paper proposes the architecture and its test-bed and prototype implementation.
\end{abstract}

Keywords: presence, activity analysis, social networks, context, prototype

\section{Introduction}

Activity analysis is an area of extensive research in domains such as healthcare, industry/workshop environments and sports. Current research focuses on lower granularities of activities where activities such as walking and running reveal information about user fitness and encourages user to be more active [8]. In this paper our focus is on inferring higher level activities which the user can share with other people, devices or services in the form of user presence. Presence is defined as the availability of a person for communication in the instant messaging (IM) domain [2]. This presence has later been extended as rich presence [11] to include other context information apart from mere availability. We state that presence extensions like RPID (RFC4480) [3] from existing PIDF (RFC 3863) [4], though a step forward, still needs further consideration. We also believe that both presence aggregation [1] which is the incorporation of presence from multiple sources (devices) and presence federation [1] which allows it to be shared across multiple technological domains (IMs, social-networking sites, etc) are also taking presence in the right direction. 
As new communication tools such as social networking sites [5] (like Facebook, LinkedIn and bookmarking sites like Delicious, Digg, StumbleUpon), blogging tools (micro-, video-, photo-, voice-blogs like Twitter, YouTube, Flickr, Bubbly respectively), citizen journalist and community news sites (Newsvine) are gaining popularity, we see a new form of communicating real-time information (relating to the user or his/her environment) in the form of status updates, tweets, etc. In this paper, we make an attempt to merge presence with activity information as we see it in the new realm of communication tools which are not limited to IM alone and include devices, services, social-networking, microblogging and community news sites. Such social interaction tools provide users with a means to communicate and share real-time information about them like activities undertaken, places visited, and the events related to the user as well as those happening in surrounding environments or which the user comes across from different media. This is a continuous process in time and space and updates are made using a number of devices from stationary (home and office) and mobile locations. This information is shared in real-time with people (family, friends, colleagues, acquaintances, complete unknowns, etc) having different types of relationships with the user. Also, we notice that the content of updates on each social networking site is related to the type of domain of that site and in turn, to the type of relationships the user has with the people in those sites. If a user is a member of the professional site LinkedIn, he/she will most likely make work-related status updates on this site and personal status updates may go to Facebook, for example if the user is writing a report and listening to music in library at the same time, he/she may want to share the information "writing a report in the library" on LinkedIn and "listening to music in library" on Facebook. Thus, to automate this process, we infuse users multiple activities to determine users multiple presence.

We believe a) that presence information is linked closely with the user's activity information, this is also evident from RFC 4480 on RPID, b) that presence is not merely linked with availability on IMs but transcends through all types of social networking tools mentioned previously, c) presence is now multi-faceted i.e. user can have different presence on different tools, and d) users can also have multiple types of presence information at the same point in time since it is linked closely to the user's activity information as mentioned in a). Thus, in this paper we present Crystal Presence (CrysP) which is the user's multiple activity infused presence information, implement the CrysP System (CrysPSys) to determine user's CrysP from their sensed multiple activity information and then intelligently share CrysP using a number of social networking tools. In section 2 , we present the related work, followed by the CrysP concept in section 3 . The architecture of CrysPSys is presented in section 4 and section 5 gives the details of the test-bed and prototype implementation. Section 6 and 7 present the discussion and conclusion and future work respectively 


\section{Related Work}

In recent years the growth of social-networking and micro-blogs have gained immense popularity and have paved the way for new means of communicating. Location is becoming an important context parameter, and geo-tagging is on the rise, for example, portals like Plazes [6] are being used for location based applications and activities by users. At the same time, there is considerable amount of research in the field of activity analysis being undertaken. An increasing number of sensors are used to detect user's physical as well as physiological activity. This research is mainly focussed in healthcare or industry environments, but we discuss some of those projects which look outside those two domains and closer to our research domain. Though, there are some similarities here with existing research but we highlight that our system looks at multiple activities of user and then its fusion with a user's multiple presence information.

In [9], the authors have built a context-aware IM client, called Nomatic IM. The main context information incorporated is location using Place Lab WLAN positioning in the campus which is then tagged by the user manually to a particular activity that they might be doing in that location. In the future the system assumes that the user will be doing the same activity at that location. CenceMe system [12], senses low level user activity information (standing/walking/running and stationary/driving are the activities sensed currently) and shares the same presence information across all social networking sites. Sensors inbuilt like the accelerometer on the Nokia N95, Nokia 5500 Sport mobile phone or the Nike+ and microphones and web cameras on the mobile phone were used. A mobile based CenceMe system where majority of the processing is done using light weight classifiers is also built. Personal Environmental Impact Report (PEIR, a Nokia funded research) [7] is an online tool to study the impact of users commuting using different types of transport and to encourage teenagers to use their bikes or public transport. A large-scale people-centric sensor network in [9] is proposed and implemented using two application scenarios: SkiScape and BikeNet. A mobile sensing platform (MSP) in [8], is a wearable device used to detect a person's activity. The system is tested in different applications like the UbiFit Garden and UbiGreen. A Micro-Blog is researched and implemented as a prototype for users in [10], which leverages localization techniques (like GPS, Wi-Fi and GSM) to spatially geo-tag user micro-blogs. The aforementioned research does not consider identifying multiple user activities simultaneously and then reason about how to share user's multiple activity information on different social networking sites.

\section{Crystal Presence}

In this section, we present the following definitions and terminology related to CrysP.

Crystal Presence (CrysP) - activity-infused, multi-faceted presence information of a person shared with the relevant devices, services and people. 
Audience - can include devices which interact with the user in different locations, services that the user might be interested in using and the user's contacts on multiple social-networking sites such as his/her family, friends and colleagues, as well as those who read the user's blogs and follow him/her on micro-blogging sites and contacts on IMs.

CrysP can be derived from the user's availability, moods, thoughts and activity. We currently focus only on activity of the user. Figure 1 shows the concept of CrysP. A user's CrysP is the multi-faceted presence information which is determined using user and environmental context information and user audience consists of everyone who is interested in this real-time information related to the user. As shown in figure 1, we can see that the user's CrysP can be of many types at different points in time and space for example, based on personal activity information such as "cooking pasta", "baking a cake", "reading a novel", "planning a vacation". Similarly, this can be based on professional activity information such as "working on research project", "writing thesis", and "reading references". Thus, we present CrysP in figure 1 which shows that, at one point in time, the user's CrysP can be a combination of these different types of activities (every facet of the crystal depicts a different type of activity at the same point in time); for example, the user can be "Cooking Pasta" and "Reading References" or "Baking a Cake" and "Watching News" or "Writing Code" and "Listening to Pink Floyd" and "Uploading images to Flickr". These activities can overlap one another. For example, we can start cooking pasta at the start of time $\left(t_{i}\right)$ $t_{1}=1 \mathrm{~min}$, and at $t_{2}=5 \mathrm{~min}$ we can be reading a reference paper, then resume to cooking pasta at $t_{3}=10 \mathrm{~min}$, at $t_{4}=12 \mathrm{~min}$ we resume reading the reference paper while the pasta cooks on till $t_{4}=20 \mathrm{~min}$. Thus, from $t_{1}=1 \mathrm{~min}$ to $t_{4}=20 \mathrm{~min}$, we were involved with two activities which were not related to each other, but overlapped each other in time slots. So, CrysP for the user will be "Cooking Pasta" and "Reading References" in a spatio-temporal domain.

The user and his/her audience interact with each other using a wide array of social-networking tools [5] (web portals like Facebook, Orkut, LinkedIn, IMs like Gtalk, Skype, micro-blogs like Twitter, etc). Table 1 shows a wide-array of tools that are available to the user to share his/her multi-faceted presence information (CrysP) with their audience. Some of these tools can be put into broad categories like, social networks, corporate social networks, social bookmarking sites, interest networks, blogs, micro-blogs, video-blogs, photo-blogs, voice-blogs, email and instant messengers. The user shares certain types of information on each of these networks depending on what type of network it is, what type of relationships he/she shares with people on that network along with what type of information related to the user people in that network are interested to see. Therefore, in this research we aim to automate the process of: a) Determining CrysP based on activity analysis and b) Share the presence and status updates with the relevant audience. Our proposed CrysP System (CrysPSys) can be seen as having the following advantages: a) it helps to reduce the amount of time people usually spend in making status updates; $3 \mathrm{~b}$ ) people who are unable to update their status information as often as they would like to, are able to 


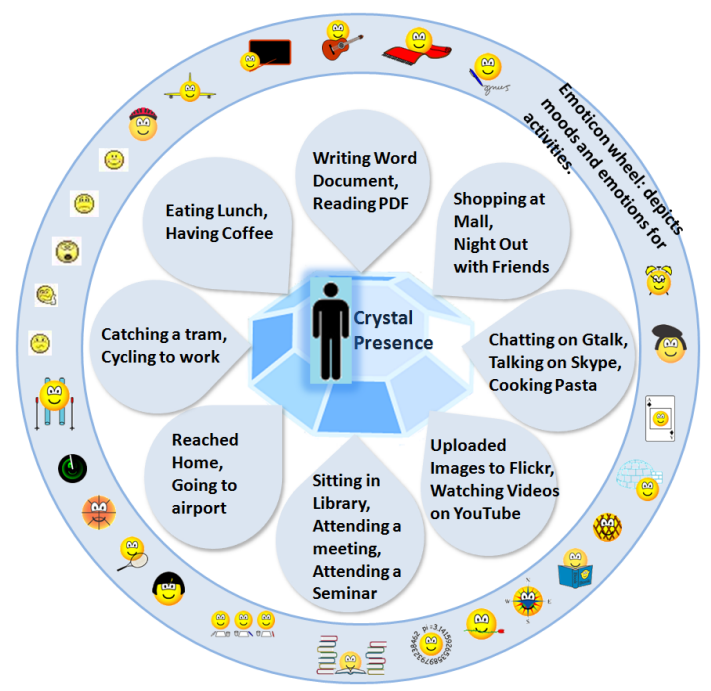

Fig. 1. Crystal Presence (CrysP).

do so via CrysPSys, c) a user can send different updates to different audience sharing only that information which is of interest to a particular set of people; $d$ ) the previous point helps in privacy concerns of the user as well; e) this provides an interesting means of passively communicating what you want to with whom you want to in real-time; f) it also opens a lot of opportunities for providing value-added services to the user based on their activities in real-time; g) it can help in reducing energy consumption in smart homes and offices as shown in scenario A below; h) such updates can also help in increasing the revenue of service providers such as telecom companies, when the updates are made from mobile devices.

Table 1. Social Network tools for sharing user's multi-faceted presence.

\begin{tabular}{ll}
\hline Types of Social Networks(SN) & Social-networks \\
\hline Personal SN & Facebook, Orkut, MySpace \\
Corporate SN & LinkedIn, Xing \\
Interest SN & HealthRanker, Newsvine, Plaxo, \\
Blogs & Blogger, Technocrati, Wordpress \\
MicroBlog (Text, Voice Video, Photo, Mood & Twitter, Tumblr, Bubbly, YouTube, \\
& TwitPic, I rate my day, Moodmill, \\
Instant Messengers & GTalk, Skype, MSN, Jaiku \\
\hline
\end{tabular}

Use case scenarios: Scenario A: John and Rhea are a couple living in one house. On a Monday, John tells Rhea that he would be working late at office. 
Rhea reaches home at 7:00PM eats her dinner and leaves John's dinner in the oven on a timer for three hours later, at 10:00PM, but John is delayed further by an hour. John's food gets heated at 10:00PM and then gets cold again. John reaches home at 11:30PM and has to reheat his food. This wastes John's time as well as electricity. If the oven could be switched on after knowing that John has reached home or when John was driving to his home, it could save the need to reheat/recook the food. Similarly, on Tuesday, Rhea was about to finish work half an hour after John, but she was unable to communicate this to him as she was in a meeting with a client. John was driving home, from work and if he was able to learn this information in real-time, John instead of going home and then returning back to pick her up, could have gone straight to Rhea's office and picked her up. If Rhea was able to share her activity information in real-time with John, this would help save John the time spent in driving again and help the couple save on their fuel costs.

Scenario B: Iris is a student at university, from the time she wakes up in the morning, till she sleeps at night, she performs a wide range of activities. She usually makes updates of random activities thoughts, moods, news/articles which she has read, study or research related documents read or experiments conducted to her social-networking sites, IM presence, micro-blog, and blog. These updates are read by a number of people which include as mentioned earlier, her family members, friends, colleagues, advisors, students, and the general public depending on where the updates are made and which tool she uses to make a particular update. This is a time consuming job and can also be distracting when you have too much work on hand. The problem of not being able to make updates and share the right information with the right audience in real time needs to be addressed. This is where our novel architecture is used to determine and detect the presence information based on activity intelligently and then share it with the audience automatically.

\section{CrysP System Architecture}

The proposed architecture is shown in Figure 2. Our architecture consists of three components, Sensing Component, CrysP Determination Component, and CrysP Sharing Component.

\subsection{Sensing Component}

The Sensing Component (SC) is responsible for collecting context information from the different wearable sensors such as accelerometers, gyroscopes, location sensors, video cameras, RFID tags, microphones, physiological sensors, and other sensors in the user's environment. Context information related to the user includes activity information and physiological information which helps to determine his/her cognitive activities as well as moods or emotions. The context information related to the user's environment is also collected such as location 
of the user (spatial context), time, day of week, date of the year (temporal context), proximity to other people, the type of social activity, etc. This context information is collected and passed on to the CrysP Determination Component where activity determination is performed.

\subsection{CrysP Determination Component}

The CrysP Determination Component (CDC) determines the user's CrysP i.e., the activity infused presence. As shown in figure 2, firstly, activities are inferred by performing segmentation and classification on the raw context information which is received from the sensors. For activity inference we use decision trees to infer body motion activities, we use RFID object interaction with temporal analysis to infer activities linked to objects. We use the Context Spaces Model [13] to infer activities at a higher level of granularity which is then further shared with any device, services or people who may be interested in this information. The pseudo code for inferring user CrysP set is as follows:

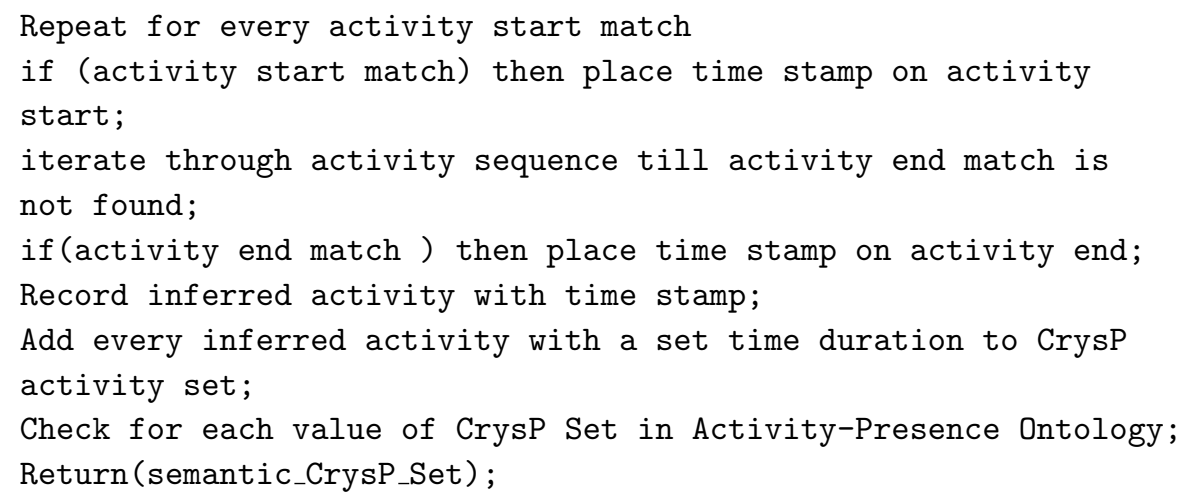

After the preliminary activity inference is done, we also determine multiple activities (level 2) based on the temporal analysis. In other words, this would also mean activities which overlap each other, or where one activity is paused to perform another activity, and the previous activity is resumed after the completion of the second activity. We only look at activities which are not related to each other i.e., they are not sub-activities of a higher level activity. The activity presence ontology is used to provide us with key sub-activities which are part of higher level activities along with the spatial and temporal aspects of each sub and higher level activity. For example, an activity like "studying in the library" can be inferred by user sitting and interacting with a word document related to his/her studies on a laptop. There can be other ways of inferring the user studying and these can be populated in the ontology. Also, if the user's location is "in library" it further adds to construct the activity "studying in library". If user is also interacting with their lunch box while sitting in the library we can add the activity "user is studying and eating food in the library". This higher level activity can then be shared others. 


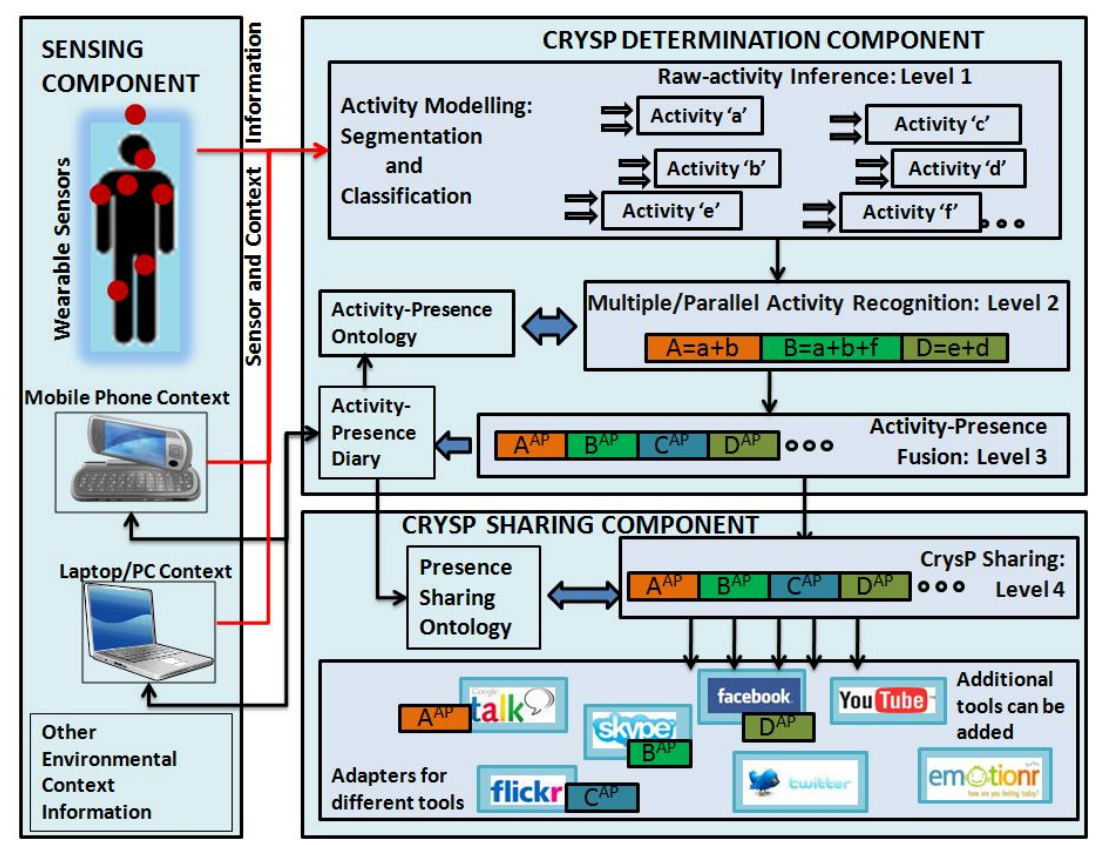

Fig. 2. Architecture of CrysP System.

In CDC, the activity-presence ontology is further used to give semantic readability to the inferred activities which creates the activity fused presence information (CrysP). This semantic meaning provides more readability as well as it removes the otherwise plain update of mere activity update, like 'walking', to 'walking to catch a tram', 'walking towards the mall', etc. The use of such ontology gives the advantage of adding more information in the way we would like to express the same activity. Here location information, distances between semantic locations and direction in which user is moving can also be used. The activity-presence diary provides the user a tool to view the CrysP information at later points in time. A user can also provide feedback to the system to help it learn and modify its behaviour, accordingly.

\subsection{CrysP Sharing Component}

In the CrysP Sharing Component (CSC), we use semantic reasoning to decide how to disseminate and share the CrysP information with the user's audience. The semantic reasoning allows for sharing the relevant CrysP information with the relevant user audience. The presence-sharing ontology is built which contains the linkages between the different social-networking tools, services and devices with the user, i.e. the type of relationship the user has with the audience. We argue that the user shares a similar class of relationship with all the contacts in each tool he/she uses in regards to the CrysP information that he/she wants to 
share using a particular tool. Though, such lines are getting blurred with social networking tools and in the future perhaps individual or group based sharing mechanisms may be required. Since, most social networks do not allow currently to share presence information in such a way and a presence update to a network is visible to all friends within that network we hereby take a homogenous approach. The CSC also includes within it a number of adapters for sharing the CrysP information with the user audience. These adapters can be added or removed based on which tools the user uses to share his/her CrysP information. Another important point to note is that the semantic rules for sharing CrysP across different tools are user-specific. Everyone can have different levels of privacy; some people are more comfortable in sharing personal information with everyone while others find it uncomfortable to do the same. Thus, the semantic rules should be easily modifiable by user intervention. The following is the pseudo code to check the type of CrysP element and then share it with the correct tool. We have built a preliminary ontology and this will be further enhanced in the future.

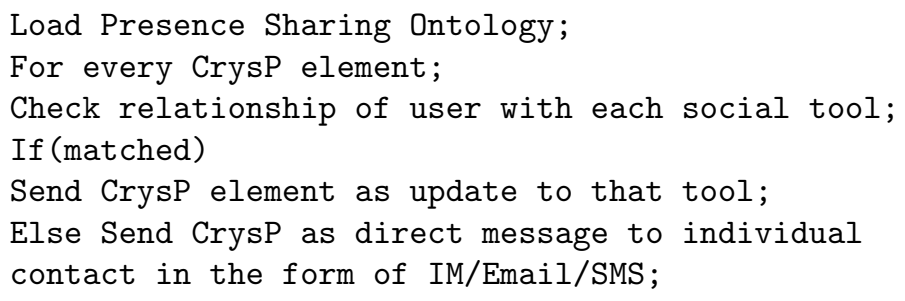

\section{Test-bed and Prototype Implementation}

We have built a test-bed comprising of several sensors such as accelerometer (body motion), indoor and outdoor positioning systems (location), temperature sensor, RFID tags and reader (object interaction) and other sophisticated software based context collection threads (user's activity on devices such as laptop and mobile phone). We place a Mulle v3 sensor (which has on it an accelerometer and a temperature sensor) on the user's waist to gather information about user body motion. Based on previous research in body motion detection using accelerometer we use chose the waist of the user as the most appropriate position [8]. The Mulle sends data at $15 \mathrm{~Hz}$ which is collected on our laptop where we use Java based Weka API to process the acceleration data. We use J48 decision tree which is an extension of C4.5 to infer three body motion related low-level activities such as sitting, standing, and walking. We achieved an accuracy of $92.56 \%{ }^{4}$. An RFID reader is attached to the user's wrist which detects RFID tagged objects such as coffee mug, lunch box, etc. The RFID reader scans for tags once every second. Each tag is labeled in terms of an activity, and the RFID readings are inferred as activities using varying time windows for different activities. This eliminates the problem of reading a tag when the object is not intentionally been touched. Multiple readings of an object over time are inferred as an intentional

\footnotetext{
${ }^{4}$ This was achieved by using a single user.
} 


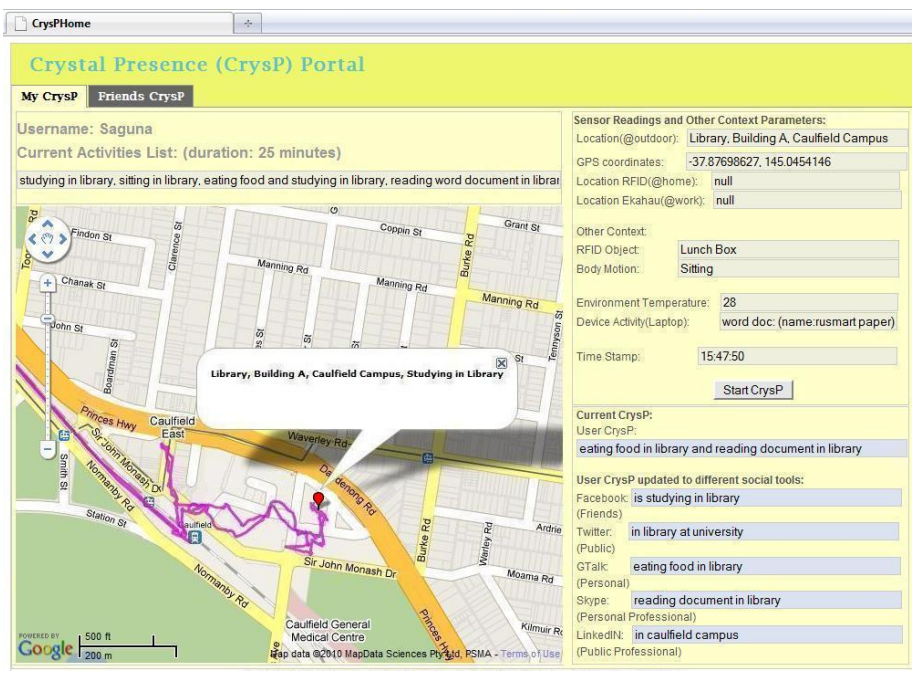

Fig. 3. CrysP Portal.

activity. The user's activity on the laptop and the mobile phone are inferred using both Java and .Net based plug-ins. A .Net based hook is used to infer the user's interaction with an application running on the device by logging mouse clicks which log current activity on the laptop such as the current application (adobe reader, word document, power point presentation, eclipse, etc). A URL logging tool Slogger is used to log URLs visited by the user in a text file and are retrieved every second to monitor user's browsing activity such as email, online news, Google search, Google scholar search, library website, airline booking site, Flickr photos, etc.

Location information is collected using three methods, GPS (outdoor), Wi-fi positioning (Ekahau WLAN positioning is deployed on university campus office of the user), and RFID based location (at home). We use the inbuilt AGPS on an android development phone (ADP1) to gather the location, speed and temperature. The RFID smart home helps in locating the user at home using multiple fixed tags. We have built a web-based GUI as shown in figure 3 to view the user's CrysP information along with the sensor/context streams. In order to share CrysP we infer activities of the user with varying time windows. The user's current location and activity can be viewed in the pop-up on the map. Also, the user's movement patterns and activities at different locations and time can be viewed by clicking anywhere on the user's path. We are further building the web-interface to make it more interactive as well as to get user feedback. We are working towards running longer experiments on a variety of users to gather different types of information. We are also building the adapters on the mobile phone to infer activity and send updates to the correct social-network/blog directly from the mobile phone.

It is also important to note that these activity updates could be shared with 
devices and services that the user uses in different locations and at different times in the day. Currently we share these updates only in the social networking domain. Adapters for sharing CrysP were written for a number of social-networking tools like LinkedIn (using OpenSocial Java API) and Facebook (using Facebook Java API); micro-blog like twitter (using Twitter Java API, twitter4j); IM like Skype (using skype_win32.jar API) and GTalk (using smack.jar API). We send CrysP updates to each of these tools depending on the preliminary presence sharing (PS) ontology. The PS ontology is written to classify the various tools and various categories of CrysP information along with the relationships between them and the user. We have only assumed five categories, personal, very personal, work, private work and public at present. We send personal updates to Facebook, very personal updates to GTalk, private work updates to Skype, public work updates to LinkedIn and public updates to Twitter. We use the Jena API to interact with the two ontologies and they are written in OWL 1.0 and RDF. Further details of the ontology are omitted here due to lack of space. Figure 4 shows how the CrysPSys shares an element of the user's CrysP with the user's Facebook audience.

\section{Discussion}

The CrysP System (CrysPSys) is currently tested in a scenario limited to a student's life. We used a specific set of sensors to infer our activities; there is a need to build the system further so that more different types of sensing technology can be incorporated into the same test-bed. There is also a requirement that labeling of activities and creating the relationships between higher and lower level activities can be easily created with limited user interaction. The higher level activity models should be built using these relationships. In our test-bed we currently inferred activities using a laptop but we intend to use the mobile phone for inference and sharing of higher level activities, though this would significantly affect the battery life of the device. The system needs to be further evaluated on multiple sets of users on longer periods of time. Further evaluation on reducing user interaction is also important.

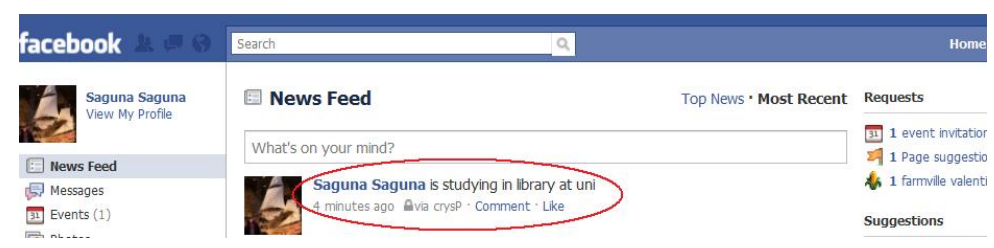

Fig. 4. Dissemination of user's CrysP to Facebook via CrysP plug-in adapter. 


\section{Conclusion and Future Work}

In this paper, we present the concept of Crystal Presence (CrysP) which opens new avenues of communicating the user's activity information with the user's audience and thus allows users to passively communicate at all times in the day. Our novel system determines activity-infused multi-faceted presence information at a point in time and each facet of this activity infused presence is shared accordingly with the intended audience. We also present a test-bed and prototype which infers multiple higher level activities of a user and then share this information with devices, services and with other people on social networking tools. In future, we will further develop and extend our architecture to incorporate more complex activities and in different scenarios with more number of users.

\section{References}

1. Jabber, The XMPP Cloud: Building a Presence-Enabled Infrastructure for RealTime Communication, White Paper, (2008)

2. RFC 2778, A Model for Presence and Instant Messaging, http://www.ietf.org/rfc/rfc2778.txt (Access Date: 12 January 2009)

3. RFC 4480, RPID: Rich Presence Extensions to the Presence Information Data Format (PIDF), http://www.rfc-editor.org/rfc/rfc4480.txt (Access Date: 12 January 2009)

4. RFC 3863, Presence Information Data Format (PIDF), http://tools.ietf.org/html/rfc3863 (Access Date: 12 January 2009)

5. Social Networking Sites, http://en.wikipedia.org/wiki/List_of_social_networking_websites, (Access Date: 12 January 2009)

6. Plazes, http://plazes.com/, (Access Date: 12 January 2009)

7. Agapie, E. et al:: Seeing our signals: Combining location traces and web-based models for personal discovery. In: HotMobile '08: Proceedings of the 9th workshop on Mobile computing systems and applications. pp. 6-10. ACM, New York, NY, USA (2008)

8. Choudhury, T. et al.: The mobile sensing platform: An embedded activity recognition system. Pervasive Computing 7(2), 32-41 (2008)

9. Eisenman, S.B. et al.: Metrosense project: People-centric sensing at scale. In: In WSW 2006 at Sensys (2006)

10. Gaonkar, S., Li, J., Choudhury, R.R., Cox, L., Schmidt, A.: Micro-blog: sharing and querying content through mobile phones and social participation. In: MobiSys '08: Proceeding of the 6th international conference on Mobile systems, applications, and services. pp. 174-186. ACM, New York, NY, USA (2008)

11. Jachner, J., Petrack, S., Darmois, E., Ozugur, T.: Rich presence: A new user communications experience. Alcatel Telecommunications Review - 1st Quarter (2005)

12. Miluzzo, E., Lane, N.D., Eisenman, S.B., Campbell, A.T.: Cenceme - injecting sensing presence into social networking applications. In: in EuroSSC, Lecture Notes in Computer Science. pp. 1-28. Springer (2007)

13. Padovitz, A., Loke, S.W., Zaslavsky, A.: Towards a theory of context spaces. In: Proceedings of the Second IEEE Annual Conference on Pervasive Computing and Communications Workshops (2004) 